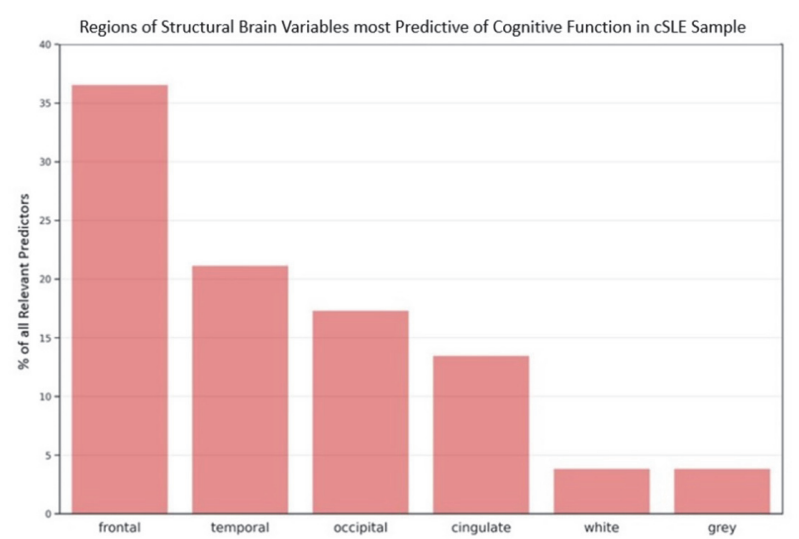

Abstract 102 Figure 1 Regions of brain variables most predictive of cognitive function in CSLE sample

brain structure variables, with the most highly associated brain measures deriving from the frontal lobe $(n=19)$, temporal lobe $(n=11)$, occipital lobe $(n=9)$ and cingulate cortex $(n=7)$. The surface area and volume of the mid-posterior corpus callosum, total left and bilateral cortical volumes, higher level of CRP and older age of patients at study visit were also found to be relevant predictors of cognitive function.

Conclusions Objective cognitive impairment was prevalent in $>40 \%$ of patients with cSLE. Impairment was strongly associated with several structural brain metrics, most of which derived from the frontal lobe. Only one disease-related factor (CRP) and one demographic factor (patient age) were found to be relevant predictors of cognitive function. Our results suggest that computational modeling has the potential to enhance diagnosis of NPSLE. Further study is needed to identify robust disease biomarkers that can be linked to functional and structural brain metrics with the use of machine learning models.

\section{NEUROPSYCHIATRIC EVENTS IN SYSTEMIC LUPUS ERYTHEMATOSUS: PREDICTORS OF OCCURRENCE}

John Hanly. Queen Elizabeth II Health Sciences Centre and Dalhousie University, Halifax, Nova Scotia, Canada

\subsection{6/lupus-2021-lupus21century.3}

Background Approximately $30-50 \%$ of neuropsychiatric (NP) events in SLE patients are attributable to lupus. Prospective studies have reported differences in the outcome of NP events depending, in part, on their attribution to SLE and non-SLE causes. The current study was performed to determine predictors for change in a patient's NP event status, based on a multistate modelling approach and attribution rules previously described.

Methods Upon enrollment and annually thereafter, NP events as per the American College of Rheumatology case definitions, were identified and attributed to SLE or non-SLE causes. Physician determined resolution was documented over time. Factors potentially associated with onset and resolution of NP events were determined by time-to-event analysis using a multistate modelling structure.

Results Over 11 years (1999 - 2011) 1,827 patients with SLE were recruited to a disease inception cohort from five different geographic areas of the world. At enrollment $88.8 \%$ were female, the mean (SD) age was 35.1 (13.3) years, and patients had variable race/ethnicity (Caucasian 48.8\%, African 16.8\%, Hispanic 15.4\%, Asian 15.1\% and other 3.9\%). The mean (SD) disease duration was 5.6 (4.2) months, SLEDAI-2K 5.3 (5.4) and SLICC/ACR damage index 0.32 (0.74). NP events occurred in 955/1,827 (52.3\%) patients and 592/1910 $(31.0 \%)$ unique events were attributed to SLE. For SLE NP events multivariate analysis revealed positive associations with male sex, concurrent non-SLE NP events excluding headache, active SLE and corticosteroids (table 1a). There was a negative

Abstract 103 Table 1a Predictors for transitions to the NP event state

\begin{tabular}{|c|c|c|c|c|}
\hline \multirow{3}{*}{$\begin{array}{l}\text { SLE NP events } \\
\text { Variable }\end{array}$} & \multicolumn{2}{|c|}{ Model 1} & \multicolumn{2}{|c|}{ Model 2} \\
\hline & \multicolumn{2}{|c|}{ ( $n=426$ transitions) } & \multicolumn{2}{|c|}{ ( $n=192$ transitions) } \\
\hline & $\mathrm{HR}(\mathrm{Cl})$ & p-value & $\mathrm{HR}(\mathrm{Cl})$ & p-value \\
\hline Male sex & $1.35(1.03,1.78)$ & 0.028 & $1.55(1.05,2.29)$ & 0.026 \\
\hline Asian race/ethnicity ${ }^{*}$ & $0.59(0.42,0.82)$ & 0.002 & $0.60(0.37,0.98)$ & 0.04 \\
\hline Post-secondary education & $0.72(0.59,0.88)$ & 0.001 & $0.73(0.55,0.98)$ & 0.040 \\
\hline Past non-SLE NP events (without headache) & $1.21(0.74,1.98)$ & 0.434 & $1.16(0.68,1.99)$ & 0.59 \\
\hline Concurrent non-SLE NP events (without headache) & $1.83(1.31,2.55)$ & $<0.001$ & $1.79(1.17,2.75)$ & 0.007 \\
\hline SLEDAI-2K (without NP variables) & & & $1.19(1.04,1.36)$ & 0.012 \\
\hline SLICC (without NP variables) & & & $1.05(0.94,1.18)$ & 0.35 \\
\hline Corticosteroids & & & $1.59(1.12,2.34)$ & 0.008 \\
\hline Anti-malarial drugs & & & $0.74(0.54,1.01)$ & 0.056 \\
\hline Immunosuppressive drugs & & & $0.67(0.50,0.94)$ & 0.019 \\
\hline \multirow[t]{2}{*}{ Non-SLE NP events excluding headaches } & \multicolumn{2}{|c|}{ Model 1} & \multicolumn{2}{|c|}{ Model 2} \\
\hline & \multicolumn{2}{|c|}{ ( $\mathrm{n}=337$ transitions) } & \multicolumn{2}{|c|}{ (not applicable) } \\
\hline Variable & $\mathrm{HR}(\mathrm{Cl})$ & p-value & & \\
\hline Non-US African race/ethnicity ${ }^{\star}$ & $0.52(0.32,0.86)$ & 0.012 & & \\
\hline Asian race/ethnicity ${ }^{*}$ & $0.40(0.26,0.62)$ & $<0.001$ & & \\
\hline Past SLE NP events & $1.29(0.84,2.00)$ & 0.24 & & \\
\hline Concurrent SLE NP events & $2.31(1.66,3.21)$ & $<0.001$ & & \\
\hline
\end{tabular}


Abstract 103 Table 1b Predictors for transitions to the resolved NP State

\begin{tabular}{|c|c|c|}
\hline \multicolumn{3}{|c|}{ SLE NP events } \\
\hline & \multicolumn{2}{|c|}{ ( $\mathrm{n}=270$ transitions) } \\
\hline Variable & $\mathrm{HR}(\mathrm{Cl})$ & p-value \\
\hline Asian race/ethnicity ${ }^{*}$ & $1.72(1.17,2.54)$ & 0.006 \\
\hline Any Peripheral & $0.89(0.65,1.24)$ & 0.500 \\
\hline Any Central-Focal & $1.74(1.33,2.27)$ & $<0.001$ \\
\hline All Central-Diffuse & 1.00 & \\
\hline Past non-SLE NP events (without headache) & $0.93(0.59,1.47)$ & 0.76 \\
\hline Concurrent non-SLE NP events (without headache) & $0.63(0.44,0.91)$ & 0.12 \\
\hline Previous headache & $0.98(0.67,1.43)$ & 0.93 \\
\hline Current headache & $0.80(0.58,1.09)$ & 0.15 \\
\hline \multicolumn{3}{|l|}{ Non-SLE NP events excluding headaches } \\
\hline & \multicolumn{2}{|c|}{ ( $\mathrm{n}=176$ transitions) } \\
\hline Variable & $\mathrm{HR}(\mathrm{Cl})$ & p-value \\
\hline Hispanic race/ethnicity* & $1.43(0.96,2.13)$ & 0.082 \\
\hline Non-US African race/ethnicity* & $2.06(1.14,3.73)$ & 0.017 \\
\hline Age at SLE diagnosis & $0.98(0.96,0.99)$ & $<0.001$ \\
\hline Any Peripheral & $0.76(0.44,1.29)$ & 0.306 \\
\hline Any Central-Focal & $1.38(0.72,2.64)$ & 0.328 \\
\hline All Central-Diffuse & 1.00 & \\
\hline
\end{tabular}

* Other race/ethnicities were included in the analysis but the results were not significant.

association with Asian race/ethnicity, post-secondary education, and immunosuppressive or anti-malarial drugs. For non-SLE NP events, excluding headache, there was a positive association with concurrent SLE NP events and negative associations with African and Asian race/ethnicity. NP events attributed to SLE had a higher resolution rate than non-SLE NP events, with the exception of headache that had comparable resolution rates. For SLE NP events, multivariate analysis revealed resolution was more common with Asian race/ethnicity and for central/focal NP events (table 1b). For non-SLE NP events resolution was more common with African race/ethnicity and less common with older age at SLE diagnosis (table 1b).

Model 1 includes time invariant variables or those defined at all time points. Model 2 is restricted to transitions for which there is information as in model 1 and additional time variable explanatory variables available only for events occurring after the initial patient assessment.

Conclusions In a large and long-term study of the occurrence and resolution of NP events in SLE we identified subgroups with better and worse prognosis. The course of NP events differs greatly depending on their nature and attribution.

Acknowledgement This work was completed by members of the Systemic Lupus International Collaborating Clinics (SLICC).

\section{DISTINCT SPATIAL PROFILE OF INFLAMMATORY GENE EXPRESSION IN THE BRAIN OF A MOUSE MODEL OF NEUROPSYCHIATRIC LUPUS} ${ }^{1,2}$ Ernest Aw, ${ }^{1}$ Yingying Zhang, ${ }^{1,2}$ Michael C Carroll*. ${ }^{1}$ Boston Children's Hospital; ${ }^{2}$ Program
in Immunology, Harvard University

\subsection{6/lupus-2021-lupus21century.4}

Background SLE is an incurable autoimmune disease that results in central nervous system (CNS) involvement with clinical manifestations including anxiety and depression. However, the mechanism/s underlying these neuropsychiatric symptoms
(NPSLE) remain unknown. An elevated type 1 interferon (IFNa) signature has been commonly observed in SLE patients, particularly within the CNS of NPSLE patients (Crow et al., 2014, Shiozawa et al., 1992). Given the diversity of clinical CNS manifestations, we hypothesized that type 1 interferonmediated inflammation occurs in spatially distinct regions within the CNS, resulting in differential behavioral outcomes depending on the impacted brain region.

Methods Spatial distribution of inflammatory gene expression in the brain was performed using MERFISH, a multiplexed spatial transcriptomics platform employing a custom set of RNA probes. Cell source and in situ results were validated by RT-PCR, RNA scope and single nucleus sequencing of RNA (sNuc-Seq).

Results Characterization of Sle 1, yaa mice in behavior assays, identified anxiety-like, and fatigue-like behaviors consistent with NPSLE. Notably, the behavior changes correlate with distinct patches of interferon stimulated gene (ISG) expression within the subcortical regions of mouse brains. Preliminary single nucleus sequencing (sNuc-Seq) and in situ hybridization results implicate astrocytes and oligodendrocytes as the major cell classes enriched in these ISG patches.

Conclusions In summary, our results validate a mouse behavioral model of NPSLE, and show spatially distinct regions of ISG expression within the CNS, opening up a new avenue of investigation into the fundamental mechanisms of NPSLE.

Acknowledgements The work was supported by a grant from the NIH R01AR072965.

\section{0 - Cutaneous SLE}

\section{TYPE I INTERFERON MODULATES LANGERHANS CELL ADAM17 IN LUPUS TO CONTRIBUTE TO PHOTOSENSITIVITY}

${ }^{1}$ Thomas M Li, ${ }^{1,2}$ Keila R Veiga, ${ }^{1,3}$ Noa Schwartz, ${ }^{4,5}$ Jose Lora, ${ }^{6}$ Ali Jabbari, ${ }^{7}$ Yong Liu, ${ }^{1,8}$ William D Shipman, ${ }^{1,9}$ Marvin J Sandoval, 'Isabel F Sollohub, ${ }^{10}$ Mehdi Rashighi, ${ }^{6} J a m e s$ G Krueger, ${ }^{7}$ Niroshana Anandasabapathy, ${ }^{11}$ David J Oliver, ${ }^{11}$ Yurii Chinenov, ${ }^{4,5}$ Carl P Blobel, 1,2,3,9Theresa T Lu*. 'Autoimmunity and Inflammation Program, HSS Research Institute, New York, NY 10021, USA; ${ }^{2}$ Pediatric Rheumatology, Department of Medicine, Hospital for Special Surgery, New York, NY, USA; ${ }^{3}$ Rheumatology, Department of Medicine, Hospital for Special Surgery, New York, NY USA; ${ }^{4}$ Arthritis and Tissue Degeneration Program, HSS Research Institute, New York, NY, USA; ${ }^{5}$ Department of Physiology, Biophysics, and Systems Biology, Weill Cornell Medicine, New York, NY, USA; ${ }^{6}$ Laboratory of Investigative Dermatology, Rockefeller University, New York, NY, USA; ${ }^{7}$ Department of Dermatology, Weill Cornell Medical College, New York, NY, USA; ${ }^{8}$ Weill Cornell TriInstitutional MD-PhD Program, New York, NY, USA; ${ }^{9}$ Department of Microbiology and Immunology, Weill Cornell Medicine, New York, NY, USA; ${ }^{10}$ Department of Dermatology, University of Massachusetts Medical School, Worcester, MA, USA; ${ }^{11}$ David Z. Rosensweig Genomics Research Center, HSS Research Institute, New York, NY, USA

\subsection{6/lupus-2021-lupus21century. 5}

Background Photosensitivity is a common feature in systemic lupus erythematosus (SLE), is considered to be a major contributor to SLE skin disease, and can be associated with serious flares of systemic disease, but mechanistic understanding remains limited. Several lines of evidence including the finding of a type I interferon (IFN-I) signature in non-lesional skin, the potentiation of keratinocyte apoptosis by IFN-I, the importance of IFNAR in murine lupus model skin lesion development, and the improved skin scores in the clinical trials of anifrolumab (anti-IFNAR1) point to a pathogenic role for IFN-I in SLE skin disease. We recently showed that Langerhans cells (LCs) limit UVR-induced keratinocyte apoptosis and skin injury via ADAM17-mediated EGFR ligand activation and 